\title{
Long-Term Variability and Outburst Activity of FS Aurigae: Further Evidence for a Third Body in the System
}

\author{
V. Neustroev ${ }^{1}$, G. Sjoberg ${ }^{2}$, G. Tovmassian ${ }^{3}$, S. Zharikov ${ }^{3}$, \\ T. Arranz Heras ${ }^{4}$, P. B. Lake ${ }^{2}$, D. Lane ${ }^{5,6}$, G. Lubcke ${ }^{2}$, and
} A. A. Henden ${ }^{2}$

${ }^{1}$ Astronomy Division, Department of Physics, P.O. Box 3000, 90014 University of Oulu, Finland. email: vitaly@neustroev.net

${ }^{2}$ American Assoc. of Variable Star Observers, 49 Bay State Road, Cambridge, MA 02138, USA

${ }^{3}$ Instituto de Astronomia, UNAM, Apdo. Postal 877, Ensenada, Baja California, 22800 Mexico

${ }^{4}$ Observatorio "Las Pegueras", Navas de Oro (Segovia), Spain

${ }^{5}$ Saint Marys University, Halifax, Nova Scotia, Canada

${ }^{6}$ The Abbey Ridge Observatory, Stillwater Lake, Nova Scotia, Canada

\begin{abstract}
FS Aurigae is famous for a variety of uncommon and puzzling periodic photometric and spectroscopic variabilities which do not fit well into any of the established sub-classes of cataclysmic variables. Here we present preliminary results of long-term monitoring of the system, conducted during the 2010-2011 observational season. We show that the long-term variability of FS Aur and the character of its outburst activity may be caused by variations in the mass transfer rate from the secondary star as the result of eccentricity modulation of a close binary orbit induced by the presence of a third body on a circumbinary orbit.
\end{abstract}

Keywords. binaries: close - novae, cataclysmic variables - stars: individual (FS Aurigae)

\section{Introduction}

FS Aurigae represents one of the most unusual cataclysmic variables (CV) to have ever been observed. The system is famous for a variety of uncommon and puzzling periodic photometric and spectroscopic variabilities which do not fit well into any of the established sub-classes of CVs (Neustroev 2002; Tovmassian et al. 2003, 2007).

Based on the short orbital period, FS Aur has been classified as a SU UMa star. Nevertheless, long-term monitoring of the system failed to detect any superoutburst in its light curve. Instead, this monitoring reveals a very long photometric period of $\sim 900$ days. Tovmassian et al. (2010) showed that such a long period may be explained by the presence of a sub-stellar third body on a circular orbit around the close binary.

In order to better understand the long-term variability and outburst activity of FS Aur, during the 2010-2011 observational season we have conducted an observing campaign, lasting more than 140 consecutive nights. Here we report the preliminary results of these observations.

\section{The 2010-2011 observing campaign}

The observations were conducted every clear night from November 26, 2010 until May 3 , 2011. The data were taken using telescopes with apertures of 0.28 to 0.5 -meters, equipped with CCD cameras and standard Johnson V filters. Depending on the weather conditions, we monitored the star for $6-8$ hours per night in the beginning of the campaign and for 3-4 hours at the end. Thus, more than 150 nights of photometry were taken 


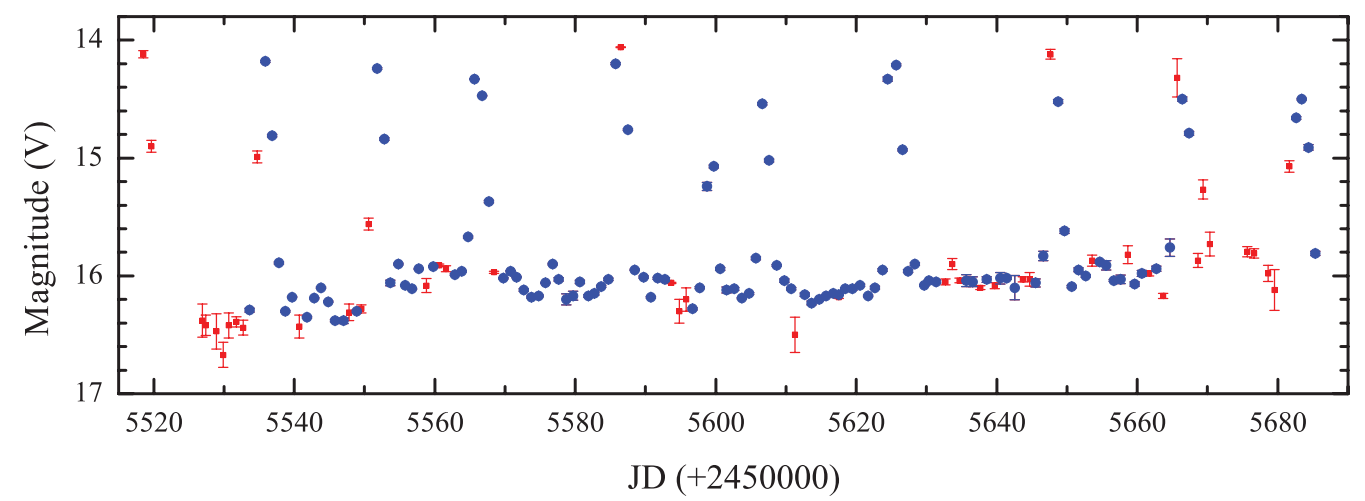

Figure 1. Light curve of FS Aur, from the 2010-2011 observing campaign. Each point is the 1-day average of observations. Blue points represent the observations obtained by the authors, while the red squares represent the AAVSO observations. Note the strong variability during the quiescent state.

(80 nights of time-resolved photometry), and almost $14000 \mathrm{~V}$-band data points were obtained. The median value of the photometric errors was $0.03 \mathrm{mag}$ in the $V$ filter. In order to reduce the scatter from both random errors and stochastic and short-term variability, we formed 1-day averages of these observations (Fig. 1). Here we note the most prominent features seen in the light curve of FS Aur: (a) during these observations, the average quiescent level increased 0.3-0.4 mag; (b) the system exhibits a strong variability even during the quiescent state; (c) in the interval between the two normal outbursts, two abnormal short low-amplitude outbursts were observed (around JD 2455600).

\section{Discussion and Conclusion}

The optical flux of a dwarf nova is dominated by the emission from an accretion disk particularly in the short period systems, while the emission from the disk is proportional to the mass-transfer rate. Schreiber et al. (2000) also found that the outburst behavior of a dwarf nova is strongly influenced by the variations of the mass-transfer rate. The latter is very sensitive to the Roche lobe size, which is proportional to the binary separation. In hierarchical triple systems, a third body can induce an eccentricity variation in an inner binary (Mazeh \& Shaham 1979; Georgakarakos 2009). The long-term modulation is produced by the time-varying tidal force of the perturber upon the binary.

Tovmassian et al. (2010) showed that a long 900-d period observed in FS Aur may be explained by the presence of a sub-stellar third body on a circular orbit around the close binary. The long-term variability of FS Aur and the character of its outburst activity may also be triggered by variations in $\dot{M}$ from the secondary as the result of eccentricity modulation of a close binary orbit induced by the presence of a third body.

\section{References}

Georgakarakos N. 2009, MNRAS, 392, 1253

Mazeh T. \& Shaham J. 1979, A\&A, 77, 145

Neustroev V. V. 2002, A\&SA, 382, 974

Schreiber M. R., Gänsicke B. T., \& Hessman F. V. 2000, A\&A, 358, 221

Tovmassian G. H., et al., 2003, PASP, 115, 725

Tovmassian G. H., Zharikov S. V., \& Neustroev V. V. 2007, ApJ, 655, 466

Tovmassian G. H., et al., 2010, arXiv:1009.5813 